\title{
THE ANOSOV THEOREM FOR INFRA-NILMANIFOLDS WITH A 2-PERFECT HOLONOMY GROUP*
}

\author{
KAREL DEKIMPE ${ }^{\dagger}$, BRAM DE ROCK $^{\ddagger}$, AND PIETER PENNINCKX ${ }^{\S}$
}

\begin{abstract}
In this paper, we show that $N(f)=|L(f)|$ for any continuous selfmap $f: M \rightarrow$ $M$ on an infra-nilmanifold $M$ of which the holonomy group is 2-perfect (i.e. having no index two subgroup). Conversely, for any finite group $F$ that is not 2-perfect, we show there exists at least one infra-nilmanifold $M$ with holonomy group $F$ and a continuous selfmap $f: M \rightarrow M$ such that $N(f) \neq|L(f)|$.
\end{abstract}

Key words. Nielsen number, Lefschetz number, infra-nilmanifold, holonomy group.

AMS subject classifications. 37C25, 54H25, 55M20.

1. Introduction. To a continuous selfmap $f: M \rightarrow M$ on a closed manifold $M$, two numbers are assigned that are of particular interest in fixed point theory: the Lefschetz number $L(f)$ and the Nielsen number $N(f)$. In [1], D. Anosov proves that $N(f)=|L(f)|$ when $M$ is a nilmanifold. He also notes that there exists a continuous selfmap $f$ on the Klein bottle such that $N(f) \neq|L(f)|$.

There are two ways to generalise the Anosov theorem. Firstly, one can search for classes of continuous selfmaps $f$ for which the equation $N(f)=|L(f)|$ holds. In [7] for example, K. Dekimpe, B. De Rock and W. Malfait prove that for an expanding selfmap $f$ on an infra-nilmanifold $M$, the equation $N(f)=|L(f)|$ holds if and only if $M$ is orientable. Additionally, the class of nowhere expanding maps on infra-nilmanifolds is introduced and the Anosov theorem is generalised to this class of maps.

Another approach is to search for classes of manifolds $M$ such that $N(f)=|L(f)|$ for every continuous selfmap $f: M \rightarrow M$. E. C. Keppelmann and C. K. McCord [11] for instance show that $N(f)=|L(f)|$ for all continuous selfmaps $f: M \rightarrow M$ on an exponential solvmanifold $M$.

For a continuous selfmap $f: M \rightarrow M$ on an arbitrary closed manifold $M$, the relation between $N(f)$ and $L(f)$ is hard to study because the Nielsen number cannot be easily calculated from its definition. In the case $M$ is an infra-nilmanifold however, K. B. Lee provides a criterion $([13])$ to decide whether $N(f)=|L(f)|$ for a given continuous selfmap $f: M \rightarrow M$. This criterion is a powerful tool in the generalisation of the Anosov theorem to certain classes of maps on infra-nilmanifolds or to certain classes of infra-nilmanifolds. For instance, it lies at the basis of the results in [7] and it is used in [8] to generalise the Anosov theorem to a well described class of infra-nilmanifolds with cyclic holonomy group.

When searching for classes of infra-nilmanifolds to which the Anosov theorem can be generalised, it is natural to use properties of the holonomy group or of the

${ }^{*}$ Received October 30, 2009; accepted for publication January 21, 2011. This research was supported by the Research Grant of the Research Foundation-Flanders (FWO): 1.5.026.05, the Research Programme of the Research Foundation-Flanders (FWO): G.0570.06, the Research Fund of the Katholieke Universiteit Leuven.

${ }^{\dagger}$ Katholieke Universiteit Leuven, Campus Kortrijk, B-8500 Kortrijk, Belgium (Karel.Dekimpe@ kuleuven-kortrijk.be).

${ }^{\ddagger}$ Katholieke Universiteit Leuven, Campus Kortrijk, B-8500 Kortrijk, Belgium. Current address: Université Libre de Bruxelles, ECARES, B-1050 Brussels, Belgium (bDeRock@ulb.ac.be).

$\S$ Katholieke Universiteit Leuven, Campus Kortrijk, B-8500 Kortrijk, Belgium (Pieter.Penninckx@ kuleuven-kortrijk.be); Current e-mail address: pieter.penninckx@scarlet.be. 
holonomy representation of an infra-nilmanifold $M$ to prove that $N(f)=|L(f)|$ for every continuous selfmap $f: M \rightarrow M$. Indeed, the Anosov theorem is originally stated for nilmanifolds, and these are precisely the infra-nilmanifolds that have trivial holonomy group. In [7], the Anosov theorem is generalised to all infra-nilmanifolds with odd order holonomy group.

In this article, we generalise the Anosov theorem to all infra-nilmanifolds of which the holonomy group is 2-perfect (i.e. having no index two subgroup). Conversely, we show that for any finite group $F$ that has an index two subgroup, there exists an infra-nilmanifold $M$ with holonomy group $F$ and a continuous selfmap $f: M \rightarrow M$ such that $N(f) \neq|L(f)|$.

The authors thank the referee for his comments.

2. Preliminaries. In this section, we introduce the basic notions needed for the formulation of the results. For more information on the Lefschetz number and the Nielsen number, we refer to $[4,10,12]$. For more information on infra-nilmanifolds, we refer to [5].

2.1. Lefschetz number and Nielsen number. Let $f: M \rightarrow M$ be a continuous selfmap on a closed manifold $M$. The Lefschetz number $L(f)$ is defined by

$$
L(f)=\sum_{i}(-1)^{i} \operatorname{Trace}\left(f_{*}: H_{i}(M, \mathbb{Q}) \rightarrow H_{i}(M, \mathbb{Q})\right) .
$$

This number is of interest in fixed point theory since $L(f) \neq 0$ implies that $f$ has a fixed point. Because the Lefschetz number is invariant under homotopy, $L(f) \neq 0$ implies that any map homotopic to $f$ has a fixed point.

Nielsen's approach to estimate the number of fixed points of $f$ is of a more geometric nature. We define an equivalence relation on the set $\operatorname{Fix}(f)$ of fixed points of $f$ : two pixed points $x, y \in \operatorname{Fix}(f)$ are $f$-equivalent if and only if there exists a path $w$ from $x$ to $y$ such that $w$ and $f \circ w$ are homotopic relative to the end points. The equivalence classes obtained this way are called fixed point classes of $f$. To each fixed point class, we assign an integer index. When this index differs from zero, we call the fixed point class essential. The Nielsen number $N(f)$ is by definition the number of essential fixed point classes of $f$. The interest in the Nielsen number arises from the fact that $N(f)$ is a lower bound for the number of fixed points of $f$. Because the Nielsen number is invariant under homotopy, every map homotopic to $f$ has at least $N(f)$ fixed points.

We say that the Anosov theorem holds for a closed manifold $M$ when $N(f)=$ $|L(f)|$ for every continuous selfmap $f: M \rightarrow M$.

2.2. Infra-nilmanifolds. Let $G$ be a connected, simply connected, nilpotent Lie group. We denote by $\operatorname{Endo}(G)$ the semigroup of all endomorphisms of $G$. The semigroup $\operatorname{Endo}(G)$ acts naturally on $G$ and contains $\operatorname{Aut}(G)$ as a subgroup. We use $\operatorname{aff}(G)$ to denote the semigroup $G \rtimes \operatorname{Endo}(G)$, which is $G \times \operatorname{Endo}(G)$ as a set, with multiplication defined by $\left(d_{1}, D_{1}\right)\left(d_{2}, D_{2}\right)=\left(d_{1} D_{1}\left(d_{2}\right), D_{1} D_{2}\right)$. An element $(d, D)$ of $\operatorname{aff}(G)$ is called an affine endomorphism of $G$ and it maps $g \in G$ to $d D(g)$. We can think of multiplication in $\operatorname{aff}(G)$ as composition of maps. When $D$ is an automorphism of $G$ (that is: $D \in \operatorname{Aut}(G)$ ), an affine endomorphism $(d, D)$ is invertible as a selfmap on $G$ and as an element of the semigroup $\operatorname{aff}(G)$. We use $\operatorname{Aff}(G)$ to denote the invertible affine endomorphisms of $G$, it equals $G \rtimes \operatorname{Aut}(G)$ and is a subgroup of the semigroup aff $(G)$. 
An almost-crystallographic group $\Gamma$ (modelled on $G$ ) is a subgroup of $\operatorname{Aff}(G)$ such that its subgroup of pure translations $N=\Gamma \cap G$ is a uniform lattice of $G$ and $N$ is of finite index in $\Gamma$. A torsion free almost-crystallographic group is an almost-Bieberbach group. We obtain an action of an almost-crystallographic group $\Gamma$ on $G$ by restricting the action of $\operatorname{Aff}(G)$ on $G$ to an action of $\Gamma$ on $G$. Then the orbit space $\Gamma \backslash G$ is compact. In the case of an almost-Bieberbach group, the orbit space $\Gamma \backslash G$ is a closed (differentiable) manifold and its fundamental group $\pi_{1}(\Gamma \backslash G)$ is isomorphic to $\Gamma$; in fact $G$ is the universal covering space of $\Gamma \backslash G$ and the group of covering transformations is exactly $\Gamma$. A manifold $M=\Gamma \backslash G$, where $\Gamma$ is an almost-Bieberbach group, is called an infra-nilmanifold (modelled on $G$ ). When $\Gamma \subset G$ is an almost-Bieberbach group, $\Gamma \backslash G$ is called a nilmanifold.

When $G=\mathbb{R}^{n}$, we refer to almost-crystallographic groups and almost-Bieberbach groups as crystallographic groups and Bieberbach groups respectively. When $\Gamma$ is a Bieberbach group, the manifold $\Gamma \backslash \mathbb{R}^{n}$ is a closed flat manifold. All closed flat manifolds can be obtained in this way.

The holonomy group $F$ of an almost-crystallographic group $\Gamma$ can be defined as the finite group

$$
F=\{x \in \operatorname{Aut}(G) \mid \exists g \in G:(g, x) \in \Gamma\} .
$$

By taking differentials, we obtain a morphism $\rho: F \rightarrow \operatorname{Aut}(\mathfrak{g})$, where $\mathfrak{g}$ is the Lie algebra associated to $G$. By fixing a basis for $\mathfrak{g}$, we obtain a faithful representation

$$
\rho: F \rightarrow \mathrm{GL}_{n}(\mathbb{R}),
$$

that we call the holonomy representation of $\Gamma$. Because of the choice of a basis, this representation is determined up to similarity.

By the holonomy group and the holonomy representation of an infra-nilmanifold, we mean the holonomy group and the holonomy representation of the associated almost-Bieberbach group.

The holonomy representation holds a lot of information about the infranilmanifold in question. For instance, the holonomy representation of an infranilmanifold determines its orientability (see [3, p. 221] and [5, p. 135]):

Proposition 2.1. Let $M$ be an infra-nilmanifold with holonomy group $F$ and holonomy representation $\rho: F \rightarrow \mathrm{GL}_{n}(\mathbb{R})$. Then $M$ is orientable if and only if $\operatorname{det}(\rho(x))=1$ for every $x \in F$.

2.3. Lefschetz number and Nielsen number of maps on infranilmanifolds. In this subsection, we present K. B. Lee's criterion to decide whether $N(f)=|L(f)|$ for a given continuous selfmap $f: M \rightarrow M$ on an infra-nilmanifold $M$. This criterion makes use of the notion of a homotopy lift.

Definition 2.2. Let $M$ be an infra-nilmanifold modelled on a connected, simply connected, nilpotent Lie group $G$. Let $f: M \rightarrow M$ be a continuous selfmap. Let $h: G \rightarrow G$ be a continuous selfmap on the universal cover $G$ of $M$. We say that $h$ is a homotopy lift of $f$ when $h$ is a lift of a map homotopic to $f$.

K. B. Lee proves the following theorem ([13, Corollary 1.2]):

THEOREM 2.3. Every continuous selfmap on an infra-nilmanifold has a homotopy lift that is an affine endomorphism. 
REMARK 2.4. Let $\Gamma$ be an almost-Bieberbach group modelled on a connected, simply connected, nilpotent Lie group $G$. Let $f: M \rightarrow M$ be a continuous selfmap on the corresponding infra-nilmanifold $M=\Gamma \backslash G$. Let $\delta \in \operatorname{aff}(G)$ be a homotopy lift of $f$. Then $f: M \rightarrow M$ induces an endomorphism $f_{\times}: \Gamma \rightarrow \Gamma$ of the covering transformation group $\Gamma$, where we consider the universal cover of $G$ over $M$. This morphism of groups $f_{\times}: \Gamma \rightarrow \Gamma$ is characterised by the equality $f_{\times}(\gamma) \delta=\delta \gamma$ for all $\gamma \in \Gamma$.

Now we have all ingredients necessary for the formulation of K. B. Lee's criterion ([13, Theorem 2.2]).

THEOREM 2.5. Let $M$ be an infra-nilmanifold modelled on a connected, simply connected, nilpotent Lie group $G$ and let $\rho: F \rightarrow \mathrm{GL}_{n}(\mathbb{R})$ be the associated holonomy representation. Let $f: M \rightarrow M$ be a continuous selfmap on $M$ and let $(d, D) \in \operatorname{aff}(G)$ be a homotopy lift of $f$. Then $N(f)=|L(f)|$ if and only if $\operatorname{det}\left(\mathbb{1}-\rho\left(x_{1}\right) D_{*}\right) \operatorname{det}(\mathbb{1}-$ $\left.\rho\left(x_{2}\right) D_{*}\right) \geq 0$ for all $x_{1}, x_{2} \in F$.

3. The Anosov theorem for infra-nilmanifolds with a 2-perfect holonomy group. Our results rely on the following basic observation.

Proposition 3.1. Let $M$ be an infra-nilmanifold modelled on a connected, simply connected, nilpotent Lie group $G$. Let $f: M \rightarrow M$ be a continuous selfmap. Let $(d, D) \in \operatorname{aff}(G)$ be a homotopy lift of $f$. Let $F$ be the holonomy group associated to $M$ and $\rho: F \rightarrow \mathrm{GL}_{n}(\mathbb{R})$ the holonomy representation. Then there exists a map $\phi: F \rightarrow F$ such that $\rho(\phi(x)) D_{*}=D_{*} \rho(x)$ for all $x \in F$.

Proof. Choose $x \in F$ arbitrarily. Choose $g \in G$ such that $(g, x)$ belongs to the almost-Bieberbach group $\Gamma$ associated to $M$. Let $f_{\times}: \Gamma \rightarrow \Gamma$ be the endomorphism of the covering transformation group $\Gamma$ induced by $f$ (where we consider the universal covering of $G$ over $M)$. Put $\left(g^{\prime}, x^{\prime}\right)=f_{\times}(g, x)$. By using Remark 2.4, one calculates that $\left(g^{\prime} x^{\prime}(d), x^{\prime} D\right)=(d D(g), D x)$, hence $x^{\prime} D=D x$. Thus for each $x \in F$, there exists $\phi(x) \in F$ such that $\phi(x) D=D x$. By taking differentials, we finish the proof of the proposition.

In the previous proposition, if $D: G \rightarrow G$ is an automorphism of Lie groups, then $\phi: F \rightarrow F$ is necessarily an isomorphism of groups. The following example illustrates that in general however, we cannot assume that $\phi: F \rightarrow F$ is a morphism of groups.

EXAMPLE 3.2. Write $F=\mathbb{Z}_{2} \oplus \mathbb{Z}_{4}$. We may identify $\mathbb{Z}_{2}$ with the matrix group generated by $-\mathbb{1}_{2}$ and $\mathbb{Z}_{4}$ with the matrix group generated by

$$
A=\left(\begin{array}{cc}
0 & 1 \\
-1 & 0
\end{array}\right)
$$

Using these identifications, we can define

$$
\begin{aligned}
& \rho_{1}: F \rightarrow \mathrm{GL}_{2}(\mathbb{R}):(x, y) \mapsto x, \\
& \rho_{2}: F \rightarrow \mathrm{GL}_{2}(\mathbb{R}):(x, y) \mapsto y \text { and } \\
& \rho_{3}: F \rightarrow \mathrm{GL}_{2}(\mathbb{R}):(x, y) \mapsto y^{2} .
\end{aligned}
$$

For $i=1, \ldots, 8$, let $e_{i} \in \mathbb{R}^{8}$ be the vector with 1 on the $i$-th place and zeroes elsewhere. Let $\Gamma$ be the Bieberbach group generated by $\mathbb{Z}^{8},\left(a_{1}, A_{1}\right)$ and $\left(a_{2}, A_{2}\right)$, 
where $a_{1}=\frac{1}{2} e_{1}+\frac{1}{2} e_{2}, a_{2}=\frac{1}{4} e_{2}$ and where $A_{1}$ and $A_{2}$ are the block diagonal matrices defined by

$$
A_{1}=\operatorname{diag}\left(\mathbb{1}_{2},-\mathbb{1}_{2}, \mathbb{1}_{2}, \mathbb{1}_{2}\right), \quad A_{2}=\operatorname{diag}\left(\mathbb{1}_{2}, \mathbb{1}_{2}, A, A^{2}\right) .
$$

Put $\rho=2 \rho_{\text {triv }} \oplus \rho_{1} \oplus \rho_{2} \oplus \rho_{3}$, then $\rho$ is the holonomy representation of $\Gamma$. Define $D$ as the blockmatrix

$$
D=\left(\begin{array}{cccc}
B & 0 & 0 & 0 \\
0 & 0 & 0 & 0 \\
0 & 0 & 0 & 0 \\
0 & \mathbb{1}_{2} & 0 & 0
\end{array}\right)
$$

where 0 indicates the $2 \times 2$ matrix consisting of zeroes and where $B=\left(\begin{array}{cc}0 & 0 \\ 1 / 2 & 0\end{array}\right) \in \mathbb{R}^{2 \times 2}$. Then

$$
\begin{aligned}
\left(a_{2}, A_{2}\right) \circ D & =D \circ\left(a_{1}, A_{1}\right), & D & =D \circ\left(a_{2}, A_{2}\right), \\
\left(a_{2}, A_{2}\right)^{2} \circ D & =D \circ\left(e_{1}, \mathbb{1}_{8}\right), & D & =D \circ\left(e_{2}, \mathbb{1}_{8}\right), \\
\left(e_{7}, \mathbb{1}_{8}\right) \circ D & =D \circ\left(e_{3}, \mathbb{1}_{8}\right), & \left(e_{8}, \mathbb{1}_{8}\right) \circ D & =D \circ\left(e_{4}, \mathbb{1}_{8}\right), \\
D & =D \circ\left(e_{5}, \mathbb{1}_{8}\right), & D & =D \circ\left(e_{6}, \mathbb{1}_{8}\right), \\
D & =D \circ\left(e_{7}, \mathbb{1}_{8}\right) \text { and } & D & =D \circ\left(e_{8}, \mathbb{1}_{8}\right),
\end{aligned}
$$

Hence $D: \mathbb{R}^{8} \rightarrow \mathbb{R}^{8}$ induces a continuous selfmap $f: \Gamma \backslash \mathbb{R}^{8} \rightarrow \Gamma \backslash \mathbb{R}^{8}$.

Suppose for a contradiction that there exists a morphism of groups $\phi: F \rightarrow F$ such that

$$
\rho(\phi(x, y)) D=D \rho(x, y) \quad \text { for all }(x, y) \in F .
$$

Write $\phi\left(-\mathbb{1}_{2}, \mathbb{1}_{2}\right)=(x, y)$, then $\rho(x, y) D=D \rho\left(-\mathbb{1}_{2}, \mathbb{1}_{2}\right)$ implies that

$$
\left(\begin{array}{cccc}
\mathbb{1}_{2} & 0 & 0 & 0 \\
0 & x & 0 & 0 \\
0 & 0 & y & 0 \\
0 & 0 & 0 & y^{2}
\end{array}\right)\left(\begin{array}{cccc}
B & 0 & 0 & 0 \\
0 & 0 & 0 & 0 \\
0 & 0 & 0 & 0 \\
0 & \mathbb{1}_{2} & 0 & 0
\end{array}\right)=\left(\begin{array}{cccc}
B & 0 & 0 & 0 \\
0 & 0 & 0 & 0 \\
0 & 0 & 0 & 0 \\
0 & \mathbb{1}_{2} & 0 & 0
\end{array}\right)\left(\begin{array}{cccc}
\mathbb{1}_{2} & 0 & 0 & 0 \\
0 & -\mathbb{1}_{2} & 0 & 0 \\
0 & 0 & \mathbb{1}_{2} & 0 \\
0 & 0 & 0 & \mathbb{1}_{2}
\end{array}\right),
$$

such that

$$
\left(\begin{array}{cccc}
B & 0 & 0 & 0 \\
0 & 0 & 0 & 0 \\
0 & 0 & 0 & 0 \\
0 & y^{2} & 0 & 0
\end{array}\right)=\left(\begin{array}{cccc}
B & 0 & 0 & 0 \\
0 & 0 & 0 & 0 \\
0 & 0 & 0 & 0 \\
0 & -\mathbb{1}_{2} & 0 & 0
\end{array}\right)
$$

such that $y^{2}=-\mathbb{1}_{2}$. Hence

$$
\begin{aligned}
&\left(\mathbb{1}_{2}, \mathbb{1}_{2}\right)=\phi\left(\mathbb{1}_{2}, \mathbb{1}_{2}\right)=\phi\left(\left(-\mathbb{1}_{2}, \mathbb{1}_{2}\right)\left(-\mathbb{1}_{2}, \mathbb{1}_{2}\right)\right) \\
&=\phi\left(-\mathbb{1}_{2}, \mathbb{1}_{2}\right) \phi\left(-\mathbb{1}_{2}, \mathbb{1}_{2}\right)=(x, y)(x, y)=\left(\mathbb{1}_{2}, y^{2}\right)=\left(\mathbb{1}_{2},-\mathbb{1}_{2}\right),
\end{aligned}
$$

a contradiction.

The observation in Proposition 3.1 is used in [7] for the proof of various theorems. One of these theorems is [7, Theorem 4.2], which can be stated as follows: 
THEOREM 3.3. Let $f: M \rightarrow M$ be an expanding selfmap on an infra-nilmanifold $M$. Then $N(f)=|L(f)|$ if and only if $M$ is orientable.

By using Proposition 3.1 and K. B. Lee's criterion, this Theorem 3.3 is a direct consequence of the following theorem:

ThEOREM 3.4. Let $\rho: F \rightarrow \mathrm{GL}_{n}(\mathbb{R})$ be a representation of a finite group $F$. Let $\phi: F \rightarrow F$ be any map. Let $D \in \mathbb{R}^{n \times n}$ be a matrix and suppose that $\rho(\phi(x)) D=$ $D \rho(x)$ for all $x \in F$. Suppose that $|\lambda|>1$ for all eigenvalues $\lambda$ of $D$. Then $\operatorname{det}(\mathbb{1}-$ $\left.\rho\left(x_{1}\right) D\right) \operatorname{det}\left(\mathbb{1}-\rho\left(x_{2}\right) D\right) \geq 0$ for all $x_{1}, x_{2} \in F$ if and only if $\operatorname{det}(\rho(x))=1$ for all $x \in F$.

In fact, this theorem is proved in the proof of Theorem 3.3 in [7].

Likewise, the proof of [7, Theorem 4.6] on nowhere-expanding maps largely consists of a proof of the following theorem:

THEOREM 3.5. Let $\rho: F \rightarrow \mathrm{GL}_{n}(\mathbb{R})$ be a representation of a finite group $F$. Let $\phi: F \rightarrow F$ be any map. Let $D \in \mathbb{R}^{n \times n}$ be a matrix and suppose that $\rho(\phi(x)) D=$ $D \rho(x)$ for all $x \in F$. Suppose that $|\lambda| \leq 1$ for all eigenvalues $\lambda$ of $D$. Then $\operatorname{det}(\mathbb{1}-$ $\left.\rho\left(x_{1}\right) D\right) \operatorname{det}\left(\mathbb{1}-\rho\left(x_{2}\right) D\right) \geq 0$ for all $x_{1}, x_{2} \in F$.

In this article, we use a similar approach. We use Proposition 3.1 and K. B. Lee's criterion (Theorem 2.5) to deduce a generalisation of the Anosov theorem from the lemma below. In this lemma we are talking about a subrepresentation $\hat{\rho}$ of a representation $\rho$. By this we mean, as usual, that there exists a subvector space $W \cong \mathbb{R}^{k}$ of $\mathbb{R}^{n}$, such that $W$ is invariant under the action of $\rho$ and the restriction of $\rho$ to $W$ is equivalent to $\hat{\rho}$. In fact, in this case there exists a second subrepresentation $\rho^{\prime}$ of $\rho$ such that $\rho$ is equivalent to $\hat{\rho} \oplus \rho^{\prime}$.

LEMMA 3.6. Let $\rho: F \rightarrow \mathrm{GL}_{n}(\mathbb{R})$ be a representation of a finite group $F$. Then the following statements are equivalent:

(A) $\operatorname{det}(\hat{\rho}(x))=1$ for all $x \in F$ and for every subrepresentation $\hat{\rho}$ of $\rho$ over $\mathbb{R}$.

(B) For any map $\phi: F \rightarrow F$ and any matrix $D \in \mathbb{R}^{n \times n}$ satisfying

$$
\rho(\phi(x)) D=D \rho(x) \quad \text { for all } x \in F,
$$

we have that

$$
\operatorname{det}\left(\mathbb{1}-\rho\left(x_{1}\right) D\right) \operatorname{det}\left(\mathbb{1}-\rho\left(x_{2}\right) D\right) \geq 0 \quad \text { for all } x_{1}, x_{2} \in F .
$$

Proof. Let us first prove the implication $(\mathbf{B}) \Longrightarrow(\mathbf{A})$. Suppose for a contradiction that statement $(\mathbf{B})$ holds, but that $\operatorname{det}(\hat{\rho}(y))=-1$ for a subrepresentation $\hat{\rho}: F \rightarrow$ $\mathrm{GL}_{\hat{n}}(\mathbb{R})$ of $\rho$ over $\mathbb{R}$ and for some $y \in F$. Choose $P \in \mathrm{GL}_{n}(\mathbb{R})$ such that $P \rho(x) P^{-1}=$ $\left(\hat{\rho} \oplus \rho^{\prime}\right)(x)$ for all $x \in F$ for a representation $\rho^{\prime}: F \rightarrow \mathrm{GL}_{n-\hat{n}}(\mathbb{R})$. By applying Theorem 3.4 with $D=2 \mathbb{1}_{\hat{n}}$, we see that there exist $x_{1}, x_{2} \in F$ such that

$$
\operatorname{det}\left(\mathbb{1}_{\hat{n}}-\hat{\rho}\left(x_{1}\right)\left(2 \mathbb{1}_{\hat{n}}\right)\right) \operatorname{det}\left(\mathbb{1}_{\hat{n}}-\hat{\rho}\left(x_{2}\right)\left(2 \mathbb{1}_{\hat{n}}\right)\right)<0 \text {. }
$$

Now put

$$
D=P^{-1}\left(\begin{array}{cl}
2 \mathbb{1}_{\hat{n}} & 0_{\hat{n} \times(n-\hat{n})} \\
0_{(n-\hat{n}) \times \hat{n}} & 0_{(n-\hat{n}) \times(n-\hat{n})}
\end{array}\right) P
$$

and let $\phi: F \rightarrow F: x \mapsto x$ be the identity map, then $\rho(\phi(x)) D=D \rho(x)$ for all $x \in F$. Additionally, $\operatorname{det}\left(\mathbb{1}-\rho\left(x_{1}\right) D\right) \operatorname{det}\left(\mathbb{1}-\rho\left(x_{2}\right) D\right)<0$, a contradiction with (B). 
Let us now prove the implication $(\mathbf{A}) \Longrightarrow(\mathbf{B})$. Suppose statement $(\mathbf{A})$ holds. Let $\phi: F \rightarrow F$ be any map and let $D \in \mathbb{R}^{n \times n}$ be a matrix such that $\rho(\phi(x)) D=D \rho(x)$ for all $x \in F$. Because $\operatorname{Ker}(D)$ is invariant under $\rho$, without loss of generality, by conjugation, we may assume that $\rho=\rho_{0} \oplus \rho_{1}$ for some representations $\rho_{0}: F \rightarrow$ $\mathrm{GL}_{n_{0}}(\mathbb{R})$ and $\rho_{1}: F \rightarrow \mathrm{GL}_{n_{1}}(\mathbb{R})$, where $n_{0}$ is the dimension of $\operatorname{Ker}(D)$ and we may assume that $D$ has the form of a blockmatrix

$$
D=\left(\begin{array}{cc}
0 & * \\
0 & D_{1}
\end{array}\right)
$$

where $D_{1} \in \mathbb{R}^{n_{1} \times n_{1}}$. Remark that for any $x \in F$,

$$
\operatorname{det}(\mathbb{1}-\rho(x) D)=\operatorname{det}\left(\mathbb{1}-\rho_{1}(x) D_{1}\right) \text { and } \rho_{1}(\phi(x)) D_{1}=D_{1} \rho_{1}(x) .
$$

Hence we may replace $\rho$ by $\rho_{1}$ and $D$ by $D_{1}$. By applying this procedure repeatedly, we may assume that $\operatorname{Ker}(D)=\{0\}$. In this case, $D$ belongs to the normaliser $N_{\mathrm{GL}_{n}(\mathbb{R})}(\rho(F))$ of $\rho(F)$ in $\mathrm{GL}_{n}(\mathbb{R})$. The centraliser $C_{\mathrm{GL}_{n}(\mathbb{R})}(\rho(F))$ of $\rho(F)$ in $\mathrm{GL}_{n}(\mathbb{R})$ is a normal subgroup of $N_{\mathrm{GL}_{n}(\mathbb{R})}(\rho(F))$ and the quotient is isomorphic to a subgroup of $\operatorname{Aut}(\rho(F))$. Since $\operatorname{Aut}(\rho(F))$ is finite, $C_{\mathrm{GL}_{n}(\mathbb{R})}(\rho(F))$ is a finite index normal subgroup of $N_{\mathrm{GL}_{n}(\mathbb{R})}(\rho(F))$ and there exists $k \in \mathbb{N} \backslash\{0\}$ such that $D^{k} \in C_{\mathrm{GL}_{n}(\mathbb{R})}(\rho(F))$.

Let $V_{\leq 1}^{\mathbb{C}}$ be the complex vector space spanned by

$\left\{z \in \mathbb{C}^{n} \mid z\right.$ is a generalised eigenvector of $D$ with eigenvalue $\lambda$, where $\left.|\lambda| \leq 1\right\}$.

Remark that the dimension of $V_{\leq 1}^{\mathbb{C}}$ equals the number of entries of modulus $\leq 1$ on the diagonal of the Jordan normal form of $D$. Since the generalised eigenspace of $D$ corresponding to an eigenvalue $\mu \in \mathbb{C}$ of $D$ is a subspace of the generalised eigenspace of $D^{k}$ corresponding to the eigenvalue $\mu^{k}$ of $D^{k}$, the vector space $V_{\leq 1}^{\mathbb{C}}$ is a subspace of the vector space spanned by

$\left\{z \in \mathbb{C}^{n} \mid z\right.$ is a generalised eigenvector of $D^{k}$ with eigenvalue $\lambda$, where $\left.|\lambda| \leq 1\right\}$.

Because these vector spaces have the same dimension, they are equal.

Let $z \in \mathbb{C}^{n}$ be in the generalised eigenspace of $D^{k}$ corresponding to an eigenvalue $\lambda \in \mathbb{C}$ of $D^{k}$ : there exists $m \in \mathbb{N} \backslash\{0\}$ such that $\left(D^{k}-\lambda \mathbb{1}\right)^{m} z=0$. Then for any $x \in F$,

$$
\left(D^{k}-\lambda \mathbb{1}\right)^{m} \rho(x) z=\rho(x)\left(D^{k}-\lambda \mathbb{1}\right)^{m} z=0,
$$

hence also $\rho(x) z$ belongs to the generalised eigenspace of $D^{k}$ that corresponds to the eigenvalue $\lambda$. Additionally,

$$
\left(D^{k}-\lambda \mathbb{1}\right)^{m} D z=D\left(D^{k}-\lambda \mathbb{1}\right)^{m} z=0,
$$

so also $D z$ is in the generalised eigenspace of $D^{k}$ that corresponds to the eigenvalue $\lambda$. We see that $V_{\leq 1}^{\mathbb{C}}$ is invariant under $\rho$ and that $D\left(V_{\leq 1}\right) \subset V_{\leq 1}$.

Similarly, one shows that the vector space $V_{>1}^{\mathbb{C}}$ spanned by

$$
\left\{z \in \mathbb{C}^{n} \mid z \text { is a generalised eigenvector of } D \text { with eigenvalue } \lambda \text {, where }|\lambda|>1\right\}
$$

is invariant under $\rho$ and that $D\left(V_{>1}^{\mathbb{C}}\right) \subset V_{>1}^{\mathbb{C}}$. Remark that $\mathbb{C}^{n}$ is the internal direct sum of $V_{\leq 1}^{\mathbb{C}}$ and $V_{>1}^{\mathbb{C}}$. Define $V_{\leq 1}=V_{\leq 1}^{\mathbb{C}} \cap \mathbb{R}^{n}$ and $V_{>1}=V_{>1}^{\mathbb{C}} \cap \mathbb{R}^{n}$. Remark that 
because $D$ is a real matrix, if $v \in V_{\leq 1}^{\mathbb{C}}$, then also the complex conjugate $\bar{v}$ belongs to $V_{\leq 1}^{\mathbb{C}}$. Hence the dimension of the real vector space $V_{\leq 1}$ equals the dimension of the complex vector space $V_{\leq 1}^{\mathbb{C}}$. Similarly, the dimension of the real vector space $V_{>1}$ equals the dimension of the complex vector space $V_{>1}^{\mathbb{C}}$. Hence $\mathbb{R}^{n}$ is the internal direct sum of $V_{\leq 1}$ and $V_{>1}$. Additionally, both $V_{\leq 1}$ and $V_{>1}$ are invariant under $\rho$ and $D\left(V_{\leq 1}\right) \subset V_{\leq 1}$ and $D\left(V_{>1}\right) \subset V_{>1}$. Hence, by conjugation, we may assume that $\rho=\rho_{<1} \oplus \rho_{>1}$ for some representations $\rho_{\leq 1}: F \rightarrow \mathrm{GL}_{n_{\leq 1}}(\mathbb{R})$ and $\rho_{>1}: F \rightarrow \mathrm{GL}_{n_{>1}}(\mathbb{R})$ and that $D$ has the form of a blockmatrix

$$
D=\left(\begin{array}{cc}
D_{\leq 1} & 0 \\
0 & D_{>1}
\end{array}\right),
$$

where $D_{\leq 1} \in \mathbb{R}^{n_{\leq 1} \times n_{\leq 1}}$ has only eigenvalues of modulus $\leq 1$ and $D_{>1} \in \mathbb{R}^{n_{>1} \times n_{>1}}$ has only eigenvalues of modulus $>1$. For any $x \in F$,

$$
\operatorname{det}(\mathbb{1}-\rho(x) D)=\operatorname{det}\left(\mathbb{1}-\rho_{\leq 1}(x) D_{\leq 1}\right) \operatorname{det}\left(\mathbb{1}-\rho_{>1}(x) D_{>1}\right) .
$$

By Theorem 3.5,

$$
\operatorname{det}\left(\mathbb{1}-\rho_{\leq 1}\left(x_{1}\right) D_{\leq 1}\right) \operatorname{det}\left(\mathbb{1}-\rho_{\leq 1}\left(x_{2}\right) D_{\leq 1}\right) \geq 0 \quad \text { for all } x_{1}, x_{2} \in F \text {. }
$$

Because $\operatorname{det}\left(\rho_{>1}(x)\right)=1$ for all $x \in F$, by Theorem 3.4,

$$
\operatorname{det}\left(\mathbb{1}-\rho_{>1}\left(x_{1}\right) D_{>1}\right) \operatorname{det}\left(\mathbb{1}-\rho_{>1}\left(x_{2}\right) D_{>1}\right) \geq 0 \quad \text { for all } x_{1}, x_{2} \in F \text {. }
$$

Hence for any $x_{1}, x_{2} \in F$,

$$
\begin{aligned}
& \operatorname{det}\left(\mathbb{1}-\rho\left(x_{1}\right) D\right) \operatorname{det}\left(\mathbb{1}-\rho\left(x_{2}\right) D\right) \\
= & \operatorname{det}\left(\mathbb{1}-\rho_{\leq 1}\left(x_{1}\right) D_{\leq 1}\right) \operatorname{det}\left(\mathbb{1}-\rho_{\leq 1}\left(x_{2}\right) D_{\leq 1}\right) \operatorname{det}\left(\mathbb{1}-\rho_{>1}\left(x_{1}\right) D_{>1}\right) \operatorname{det}\left(\mathbb{1}-\rho_{>1}\left(x_{2}\right) D_{>1}\right) \\
\geq & 0 .
\end{aligned}
$$

Using Proposition 3.1 and K. B. Lee's criterion (Theorem 2.5), we 'translate' this lemma into a generalisation of the Anosov theorem:

THEOREM 3.7. Let $M$ be an infra-nilmanifold with holonomy group $F$ and holonomy representation $\rho: F \rightarrow \mathrm{GL}_{n}(\mathbb{R})$. Suppose that $\operatorname{det}(\hat{\rho}(x))=1$ for all $x \in F$ and for every subrepresentation $\hat{\rho}$ of $\rho$ over $\mathbb{R}$. Then the Anosov theorem holds for $M$.

Proof. Let $G$ be the connected, simply connected, nilpotent Lie group on which $M$ is modelled. Let $f: M \rightarrow M$ be a continuous selfmap and let $(d, D) \in \operatorname{aff}(G)$ be a homotopy lift of $f$. By Proposition 3.1, there exists a map $\phi: F \rightarrow F$ such that $\rho(\phi(x)) D_{*}=D_{*} \rho(x)$ for all $x \in F$. By Lemma 3.6,

$$
\operatorname{det}\left(\mathbb{1}-\rho\left(x_{1}\right) D_{*}\right) \operatorname{det}\left(\mathbb{1}-\rho\left(x_{2}\right) D_{*}\right) \geq 0 \quad \text { for all } x_{1}, x_{2} \in F .
$$

By Theorem 2.5, $N(f)=|L(f)|$.

Remark that for a representation $\rho: F \rightarrow \mathrm{GL}_{n}(\mathbb{R})$ of a finite cyclic group $F$, statement (A) in Lemma 3.6 is equivalent to the condition that -1 is no eigenvalue of $\rho\left(x_{0}\right)$, where $x_{0}$ is a generator of $F$. This is precisely the condition on the holonomy representation of an infra-nilmanifold $M$ with cyclic holonomy group under which the 
main result of [8] states that the Anosov theorem holds for $M$. Hence Theorem 3.7 generalises the main result of [8].

Let us now state and prove the main theorem of this section:

TheOrem 3.8. Let $F$ be a finite group. Then the Anosov theorem holds for every infra-nilmanifold with holonomy group $F$ if and only if $F$ is 2-perfect.

Proof. Suppose $F$ has no index two subgroup. Let $M$ be an infra-nilmanifold with holonomy group $F$ and holonomy representation $\rho: F \rightarrow \mathrm{GL}_{n}(\mathbb{R})$. We want to show that the Anosov theorem holds for $M$. By Theorem 3.7, it suffices to show that $\operatorname{det}(\hat{\rho}(x))=1$ for all $x \in F$ and for every subrepresentation $\hat{\rho}$ of $\rho$ over $\mathbb{R}$. Suppose for a contradiction that there exists a subrepresentation $\hat{\rho}$ of $\rho$ over $\mathbb{R}$ and an element $y$ of $F$ such that $\operatorname{det}(\hat{\rho}(y))=-1$. Define

$$
\psi: F \rightarrow\{-1,1\}: x \mapsto \operatorname{det}(\hat{\rho}(x)),
$$

then $\psi^{-1}(\{1\})$ is an index two subgroup of $F$, a contradiction. Hence $\operatorname{det}(\hat{\rho}(x))=1$ for all $x \in F$ and for every subrepresentation $\hat{\rho}$ of $\rho$ over $\mathbb{R}$. By Theorem 3.7, the Anosov theorem holds for $M$.

Conversely suppose that $F$ has an index two subgroup $H$. We want to show there exists an infra-nilmanifold $M$ with holonomy group $F$ and a continuous selfmap $f: M \rightarrow M$ such that $N(f) \neq|L(f)|$. By [2, Theorem 3], there exists a closed flat manifold $M$ with holonomy group $F$. We consider two cases.

Suppose $M$ is not orientable. By [9], there exists an expanding map $f: M \rightarrow M$. By Theorem 3.4, $N(f) \neq|L(f)|$.

Suppose $M$ is orientable. Let $\Gamma \subset \operatorname{Aff}\left(\mathbb{R}^{n}\right)$ be the Bieberbach group associated to $M$ and $\rho: F \rightarrow \mathrm{GL}_{n}(\mathbb{R})$ the holonomy representation. Define the representation

$$
\hat{\rho}: F \rightarrow \mathrm{GL}_{1}(\mathbb{R}): x \mapsto \begin{cases}1 & \text { if } x \text { belongs to the index two subgroup } H \text { of } F \\ -1 & \text { else. }\end{cases}
$$

Now $\Gamma$ acts on $\mathbb{Z}$ by

$$
(a, A) \cdot z=\hat{\rho}(A)(z) \quad \text { for every }(a, A) \in \Gamma \text { and every } z \in \mathbb{Z} \text {. }
$$

Define $\Gamma^{\prime}$ as the semi-direct product $\Gamma^{\prime}=\mathbb{Z} \rtimes \Gamma$. Because $\Gamma$ is torsion free, also $\Gamma^{\prime}=$ $\mathbb{Z} \rtimes \Gamma$ is torsion free. We can embed $\Gamma^{\prime}$ in $\operatorname{Aff}\left(\mathbb{R}^{n+1}\right)$ by identifying $(z,(a, A)) \in \Gamma^{\prime}$ with $(t,(\hat{\rho} \oplus \rho)(A)) \in \operatorname{Aff}\left(\mathbb{R}^{n+1}\right)$, where $t=\left(z, a_{1}, \ldots, a_{n}\right)$ if $a=\left(a_{1}, \ldots, a_{n}\right) \in \mathbb{R}^{n}$. Because $\Gamma \cap \mathbb{R}^{n}$ is a uniform lattice in $\mathbb{R}^{n}$, also $\Gamma^{\prime} \cap \mathbb{R}^{n+1}$ is a uniform lattice in $\mathbb{R}^{n+1}$. Let $\left\{\alpha_{1}, \ldots, \alpha_{m}\right\}$ be a finite set of representatives of the right cosets of $\Gamma \cap \mathbb{R}^{n}$ in $\Gamma$. Because every $(z,(a, A)) \in \Gamma^{\prime}$ can be written as $(z,(0, \mathbb{1}))(0,(a, A))=(z,(0, \mathbb{1}))(0,(t, \mathbb{1}))\left(0, \alpha_{i}\right)$ for some $t \in \Gamma \cap \mathbb{R}^{n}$ and some $\alpha_{i}$ and because $(z,(0, \mathbb{1}))(0,(t, \mathbb{1})) \in \Gamma^{\prime} \cap \mathbb{R}^{n+1}$, we see that $\Gamma^{\prime} \cap \mathbb{R}^{n+1}$ has only finitely many right cosets. Hence $\Gamma^{\prime} \cap \mathbb{R}^{n+1}$ is of finite index in $\Gamma^{\prime}$ and hence $\Gamma^{\prime}$ is a Bieberbach group. Let $M^{\prime}$ be the associated closed flat manifold. It is clear that $F$ is the holonomy group of $M^{\prime}$ and that $\hat{\rho} \oplus \rho$ is the holonomy representation. Again, we can find an expanding map $f^{\prime}: M^{\prime} \rightarrow M^{\prime}$ and because $M^{\prime}$ is not orientable by Proposition 2.1, we have that $N\left(f^{\prime}\right) \neq\left|L\left(f^{\prime}\right)\right|$ by Theorem 3.4. ㅁ

Corollary 3.9. As a special case of Theorem 3.8, the Anosov theorem holds for any infra-nilmanifold of which the holonomy group is a simple group, different 
from $\mathbb{Z}_{2}$ (the group with two elements). This is a new result and does not follow from earlier publications.

REMARK 3.10. When the holonomy group $F$ of an infra-nilmanifold $M$ has an index two subgroup, this does not a priori imply there exists a continuous selfmap $f: M \rightarrow M$ for which $N(f) \neq|L(f)|$. A generalised Hantzsche-Wendt manifold is by definition an $n$-dimensional closed flat manifold with holonomy group isomorphic to $\mathbb{Z}_{2}^{n-1}$. The groups $\mathbb{Z}_{2}^{n-1}$ clearly have an index two subgroup (at least when $n>$ 1). Nevertheless, the Anosov theorem holds for all orientable generalised HantzscheWendt manifolds $([6])$.

REMARK 3.11. Whether the Anosov theorem holds for an infra-nilmanifold $M$ does not depend solely on the holonomy representation of $M$. For instance, let $\Gamma$ be the Bieberbach group generated by $\left(e_{1}, \mathbb{1}_{4}\right),\left(e_{2}, \mathbb{1}_{4}\right),\left(e_{3}, \mathbb{1}_{4}\right),\left(e_{4}, \mathbb{1}_{4}\right),\left(a_{1}, A_{1}\right)$ and $\left(a_{2}, A_{2}\right)$, where $e_{i} \in \mathbb{R}^{4}$ has 1 on the $i$-th place and 0 elsewhere, $a_{1}=\left(0, \frac{1}{2}, \frac{1}{2}, 0\right), a_{2}=$ $\left(0,0,0, \frac{1}{2}\right), A_{1}=\operatorname{diag}(1,1,-1,-1)$ and $A_{2}=\operatorname{diag}(1,-1,-1,1)$. Let $M$ be the closed flat manifold associated to $\Gamma$. One can verify by using Theorem 2.5 that $N(f)=|L(f)|$ for every continuous selfmap $f: M \rightarrow M$. Let $\Gamma^{\prime}$ be the Bieberbach group generated by $\left(e_{1}, \mathbb{1}_{4}\right),\left(e_{2}, \mathbb{1}_{4}\right),\left(e_{3}, \mathbb{1}_{4}\right),\left(e_{4}, \mathbb{1}_{4}\right),\left(a_{1}^{\prime}, A_{1}\right)$ and $\left(a_{2}, A_{2}\right)$, where $a_{1}^{\prime}=\left(\frac{1}{2}, 0,0,0\right)$. Let $M^{\prime}$ be the closed flat manifold associated to $\Gamma^{\prime}$. Put $d=(0,0,0,0)$ and let $D$ be the matrix $D=\operatorname{diag}(3,0,3,3)$, then $\operatorname{det}\left(\mathbb{1}_{4}-D\right) \operatorname{det}\left(\mathbb{1}_{4}-A_{1} D\right)<0$. One can verify that $(d, D) \in \operatorname{aff}\left(\mathbb{R}^{4}\right)$ induces a continuous selfmap $f^{\prime}: M^{\prime} \rightarrow M^{\prime}$ and by Theorem 2.5, $N\left(f^{\prime}\right) \neq\left|L\left(f^{\prime}\right)\right|$. Remark that $M$ and $M^{\prime}$ have the same holonomy group $\mathbb{Z}_{2}^{2}$ and the same holonomy representation.

\section{REFERENCES}

[1] D. Anosov, The Nielsen numbers of maps of nil-manifolds, Uspekhi. Mat. Nauk, $404(224)$ (1985), pp. 133-134. English transl.: Russian Math. Surveys, 40:4 (1985), pp. 149-150.

[2] L. Auslander And M. Kuranishi, On the holonomy group of locally Euclidean spaces, Ann. of Math. (2), 65 (1957), pp. 411-415.

[3] K. S. Brown, Cohomology of groups., volume 87 of "Grad. Texts in Math.", Springer-Verlag New York Inc., 1982.

[4] R. F. Brown, The Lefschetz fixed point theorem, Scott, Foresman and Company, 1971.

[5] K. Dekimpe, Almost-Bieberbach Groups: Affine and Polynomial Structures, volume 1639 of "Lect. Notes in Math.", Springer-Verlag, 1996.

[6] K. Dekimpe, B. De Rock, and W. Malfait, The Anosov theorem for flat generalized Hantzsche-Wendt manifolds, J. Geom. Phys., 52 (2004), pp. 174-185.

[7] K. Dekimpe, B. De Rock, and W. Malfait, The Anosov relation for Nielsen numbers of maps of infra-nilmanifolds, Monatschefte für Mathematik, 150 (2007), pp. 1-10.

[8] K. Dekimpe, B. De Rock, And W. Malfait, The Anosov theorem for infra-nilmanifolds with cyclic holonomy group, Pacific J. Math., 229:1 (2007), pp. 137-160.

[9] D. Epstein And M. Shub, Expanding endomorphisms of flat manifolds, Topology, 7 (1968), pp. $139-141$.

[10] B. JiAng, Nielsen Fixed Point Theory, volume 14 of "Contemp. Math.", American Mathematical Society, 1983.

[11] E. C. Keppelmann And C. K. McCord, The Anosov theorem for exponential solvmanifolds, Pacific J. Math., 170:1 (1995), pp. 143-159.

[12] T.-H. KIANG, The Theory of Fixed Point Classes, Springer-Verlag, 1989.

[13] K. B. LEE, Maps on infra-nilmanifolds, Pacific J. Math., 168:1 (1995), pp. 157-166. 